\title{
Coherent De-dispersion Observations at Jodrell Bank
}

\author{
S. M. Ord, 1. H. Stairs and F. Camilo \\ University of Manchester, Jodrell Bank Observatory, Cheshire, U.K., \\ $S K 119 D L$
}

\begin{abstract}
.
Coherent de-dispersion removes the dispersive effect of the interstellar medium by convolving the digitally sampled telescope output voltages with an inverse filter function, derived from the tenuous plasma dispersion law. The time resolution obtainable by this method is limited only by the bandwidth that can be sampled sufficiently fast as to avoid aliasing. This allows high time resolution, therefore high precision, timing and polarimetry observations of millisecond pulsars. We present here the first results from the Jodrell Bank coherent de-dispersion system.
\end{abstract}

\section{Introduction}

Coherent de-dispersion removes the dispersive broadening by convolving the data with the inverse I.S.M. transfer function. The resulting de-dispersed data stream is then detected and folded at the pulsar period and integrated to produce a pulse profile.

As the whole bandpass is processed the time resolution is given by $1 / \mathrm{B}$, where $\mathrm{B}$ is the bandwidth. This is a considerable improvement over contemporary filterbank systems.

\section{Acquisition and Processing}

The Jodrell Bank coherent de-dispersion system is based upon the Princeton Mark 4 system which is installed at Arecibo Observatory. The observing system consists of a set of filters, amplifiers and digital samplers, the purpose of which is to sample the raw voltages induced in the receiver by the radio signals. At present the Jodrell Bank system has a maximum bandwidth of $10 \mathrm{MHz}$ (going up to $20 \mathrm{MHz}$ in the near future) and can sample with either 2 or 4 bit digitisation.

The off-line processing of the observations is performed on a 576 processor CRAY T3E, which is housed at the University of Manchester Computer Centre. Access to this machine is provided by Computer Services for Academic Research (C.S.A.R.).

The processing of the data follows three main steps. Firstly the recorded data is read from a DLT and each processor is given access to a section of this data. The next step is the de-dispersion itself which is performed independently on each processor. The final step is the recombination of the output from each processor to produce a single file containing all of the integrations. 

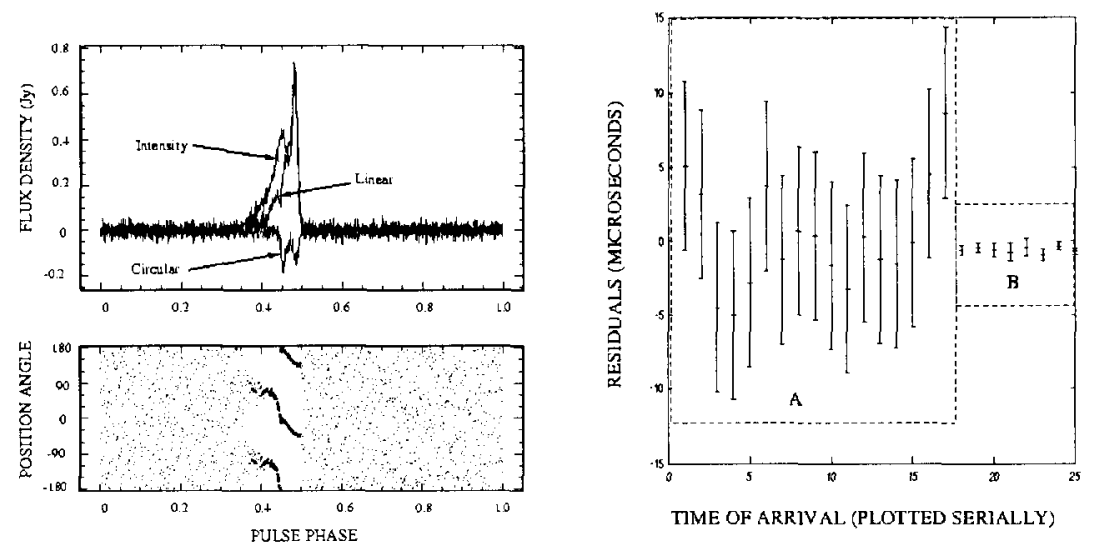

Figure 1. The left panels show the flux calibrated pulse profile together with linear and circular polarization levels of $\mathrm{J} 1022+1001$, a binary millisecond pulsar with a spin period of $16 \mathrm{~ms}$ and an orbital period of 7.8 days. The observations were taken at $610.5 \mathrm{MHz}$. The right panel shows the residuals from a fit of pulse times of arrival to a theoretical model of the behavior of B1937+21. TOAs obtained with the Jodrell Bank filterbank are shown in area A. Area B contains TOAs obtained with the coherent de-dispersion system. The improvement in timing accuracy is evident.

\section{Observations}

The output file can be analysed to provide information about polarization, the structure of pulse profiles at very high time resolution and of course very accurate timing studies can be performed. Some sample observations are presented in Figure 1.

\section{Future Work}

We plan to use this observing system to not only study the timing behaviour and polarimetry of millisecond pulsars but to also examine the giant pulses displayed by both B1937+21 and B0531+21, the Crab pulsar. We will also search, with high time resolution, for pulsars in globular clusters.

Over the next twelve months we intend to expand the observing bandwidth of this system by a factor of two, which will increase the sensitivity of the instrument at the expense of doubling the data rate and extending the processing time.

The flexibility of the observing system together with the processing power of the CRAY T3E will make this expansion relatively simple to perform. 